\title{
Arts, Design, Entertainment, Sports and Media Occupations
}

National Cancer Institute

\section{Source}

National Cancer Institute. Arts, Design, Entertainment, Sports and Media Occupations. NCI Thesaurus. Code C97625.

A class of professional or vocational positions of employment that involve arts, design, entertainment, sports or media. 